\title{
Bronchoscopic Transparenchymal Nodule Access: Feasibility and Safety in an Endoscopic Unit
}

\author{
Dominik Harzheim ${ }^{a}$ Daniel Sterman ${ }^{c}$ Pallav L. Shah ${ }^{d-f}$ Ralf Eberhardt ${ }^{a, b}$ \\ Felix J.F. Herth ${ }^{a, b}$ \\ a Pneumology and Respiratory Care Medicine, and ${ }^{b}$ Translational Lung Research Center Heidelberg (TLRCH), \\ German Center for Lung Research (DZL), University of Heidelberg, Heidelberg, Germany; 'Department of Pulmonary \\ Medicine, New York, N.Y., USA; ${ }^{d}$ The NIHR Respiratory Biomedical Research Unit at Royal Brompton and Harefield \\ NHS Foundation Trust and Imperial College, ${ }^{e}$ Chelsea and Westminster Hospital, and ${ }^{\mathrm{f}}$ National Heart and Lung \\ Institute, Imperial College, London, UK
}

\section{Key Words}

Lung cancer · Bronchoscopy · Non-small-cell lung cancer · Solitary pulmonary nodules · Lung masses ·

Transparenchymal biopsy

\begin{abstract}
Background: The minimal invasive investigation of solitary pulmonary nodules becomes increasingly important with the emergence of lung cancer screening. Objectives: We report the results of the first utilization of a recently developed procedure in a bronchoscopy suite, which approaches solitary pulmonary nodules via a transparenchymal path. Methods: This study was a prospective, single-arm interventional study. We investigated patients with a solitary pulmonary nodule detected on CT imaging, which was suspicious for malignancy. The subject's CT was employed to calculate an airway wall point of entry (POE) as well as an avascular path through lung tissue from the POE to the solitary pulmonary nodule. Using a set of catheter-based tools under fused fluoroscopy guidance, a tunnelled tract was created from the POE to the nodule. The patients were surveyed for at least
\end{abstract}

\section{KARGER}

E-Mail karger@karger.com www.karger.com/res
$72 \mathrm{~h}$ in our hospital. The primary end point of the study was to evaluate the feasibility to access and biopsy solitary pulmonary nodules outside of an operation theatre. Results: Six patients were recruited, and a tunnel pathway was created in 5 patients. There were no adverse events during the procedures. Two pneumothoraces were diagnosed by chest $\mathrm{X}$ ray $2 \mathrm{~h}$ after the procedure, with one pneumothorax requiring drainage. Adequate biopsies were obtained from all 5 patients in whom a tunnel path was created. Conclusions: This study demonstrates that bronchoscopic transparenchymal access of solitary pulmonary nodules is feasible outside an operation theatre.

(c) 2016 S. Karger AG, Basel

\section{Introduction}

Lung cancer constitutes the most commonly diagnosed cancer and leading cause of cancer death worldwide $[1,2]$. Despite perpetual investigational efforts, lung cancer is still associated with a poor prognosis, accompanied by a 5 -year survival rate of $9 \%$ [1]. With delayed de-
(C) 2016 S. Karger AG, Basel

0025-7931/16/0914-0302\$39.50/0 
velopment of symptoms, most lung cancers are classified as advanced at the time of diagnosis (40\% stage IV, $30 \%$ stage III), resulting in the above-mentioned poor prognosis. A promising step towards improvement in lung cancer prognosis was the indication of a $20 \%$ reduction in lung cancer mortality by chest CT screening, suggested by the National Lung Screening Trial that examined 53,454 persons at high risk for lung cancer [3].

However, $27.3 \%$ of patients undergoing the screening trial had at least one pulmonary nodule requiring further diagnostic follow-up. This included 713 (4\%) surgical procedures, resulting in 165 cases with at least one complication, associated with 5 deaths. Surgical diagnosis of screening-detected nodules deteriorates the value of screening through cost implications and risks of surgical procedures [4]. Alternatives to the surgical approach are transthoracic procedures and bronchoscopic techniques. The former have a higher diagnostic accuracy at the cost of a significant complication profile with a pneumothorax rate of $15 \%$ [5]. Bronchoscopy, with a risk of $<3 \%$ for pneumothoraces, constitutes a less invasive approach. However, the conventional bronchoscopic approach with fluoroscopic guidance of transbronchial biopsies yields low sensitivity scores for peripheral nodules [6], especially in lesions $<2 \mathrm{~cm}$.

Due to its low sensitivity, a bronchoscopic diagnosis of small pulmonary nodules was formerly not recommended by the American College of Chest Physicians [7]. With the emergence of bronchoscopic guidance techniques, this statement was revised, taking the higher diagnostic yield of navigation systems such as endobronchial ultrasound, ultrathin bronchoscopy, virtual bronchoscopy and electromagnetic navigation into account [8]. Through these methods, the diagnostic yield regarding malignant nodules measuring $\leq 2 \mathrm{~cm}$ could be raised from a prior reported overall sensitivity of $33 \%$ by fluoroscopy-guided bronchoscopy to a pooled diagnostic yield of $61 \%[9,10]$. The yield varies depending on factors such as the nodule diameter, its distance from the hilum and the so-called 'bronchus sign' [11]. When there is no visible bronchus leading to the lesion, the probability of reaching the nodule by a transbronchial biopsy is low. Besides, navigation through multiple subsegments is challenging, decreasing the diagnostic yield with every branch beyond the fourth generation of the bronchial tree.

We have further evaluated the feasibility and safety of the newly developed Bronchoscopic Transparenchymal Nodule Access (BTPNA) procedure in the setting of a bronchoscopy suite.

Bronchoscopic Transparenchymal Nodule Access

\section{Methods}

We conducted a prospective, single-arm interventional study by recruiting patients with pulmonary nodules, which are defined as lesions of a diameter up to $30 \mathrm{~mm}$. A 'conventional' transbronchial approach appeared unpromising due to the peripheral location of the nodules and the missing bronchus sign. Participants were required to fulfill all the inclusion criteria in the absence of any exclusion criteria and provide written informed consent. The aim of this study was to demonstrate the safe use of BTPNA outside of an operation theatre. Patients were recruited from April 2014 to October 2014. The study was approved by the Ethics Committee of the University of Heidelberg (CIV 1210009122).

For detailed information regarding patient selection, planning stage and procedure, see also Herth et al. [12].

\section{Planning Stage and Procedure}

The Archimedes Virtual Bronchoscopy Navigation System (Broncus Medical, Mountain View, Calif., USA) was used to reconstruct CT data of eligible subjects into a $3 \mathrm{D}$ model which provided guidance of a sheath from the POE on the airway wall through the lung parenchyma directly to the solitary pulmonary nodule (fig. 1, 2). The procedure planning time depicts the work with the Archimedes Virtual Bronchoscopy Navigation System prior to the bronchoscopic procedure. It contains the selection of the exact point of entry (POE) location, the adaption of the tunnel path as well as the familiarization with the anatomical features of the bronchoscopic path to the lung nodule.

The bronchoscopic procedure was performed under general anaesthesia and muscle paralysis in our bronchoscopy suite. The procedures were performed by F.J.F.H. and R.E., who had used the system in the earlier feasibility study [12].

After the POE location was reached under virtual guidance, the subsequent procedural steps were similar to those performed in the feasibility study [12]. A coring needle (FleXNeedle; Broncus Medical) was inserted through the working channel of a standard therapeutic bronchoscope (1T180; Olympus Medical, Tokyo, Japan) and through the airway wall at the identified POE in the airway. Once the sheath with radiopaque marker bands and a blunt dissection stylet created a tunnel within the lung parenchyma to the proximity of the nodule, samples were taken by biopsy forceps under fused fluoroscopic guidance.

Two hours after the BTPNA procedure, a chest X-ray was performed, and the patient was surveyed for at least $72 \mathrm{~h}$ in our hospital. Complications, if any, were recorded. Operative resection was performed after a mean of 14 days.

\section{Outcomes}

The primary objective of the study was to further evaluate the feasibility and safety of the BTPNA procedure in a standard bronchoscopy suite. Safety was evaluated by assessing any intraprocedural complications, such as localized bleeding or cardiopulmonary instability and postprocedural complications such as the development of a pneumothorax. The subjects were monitored for at least $72 \mathrm{~h}$ after the procedures, including a chest X-ray in order to rule out late complications related to the BTPNA. The severity of adverse events was defined according to the ISO 14155:2011 definition.

Feasibility was defined as the proportion of subjects where the procedure could be completed with obtainment of at least one biopsy sufficient for a histological diagnosis. The histology attained 


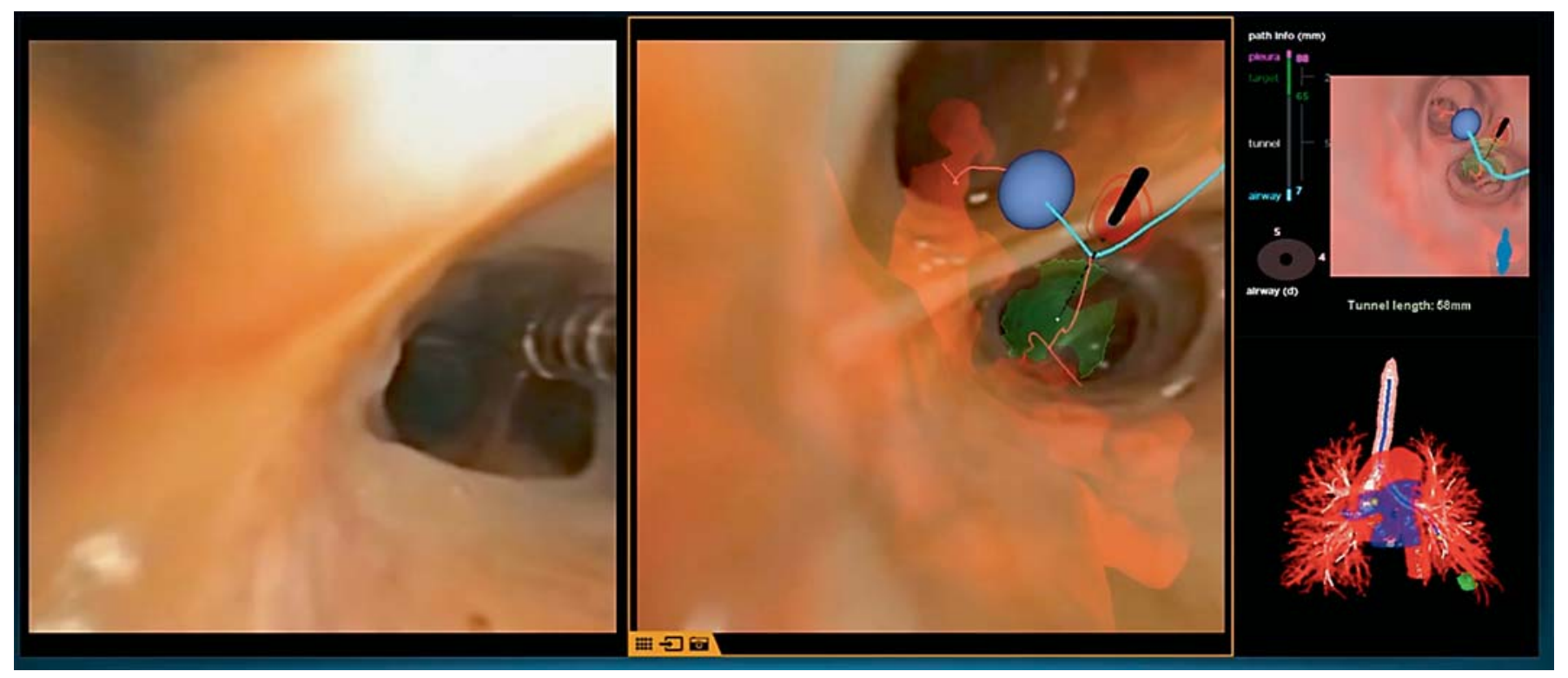

Fig. 1. Penetration of the airway wall at the POE under Archimedes Virtual Bronchoscopy Navigation guidance.

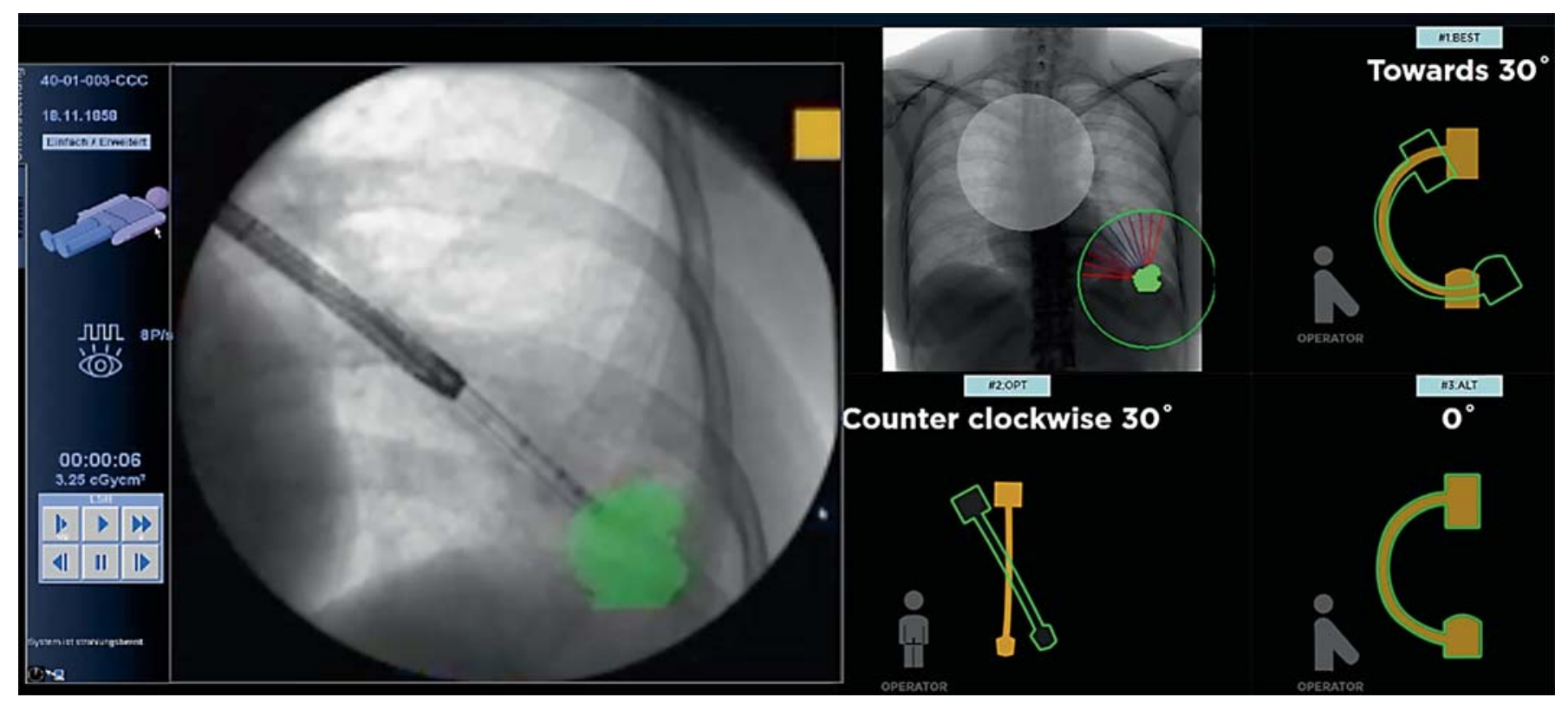

Fig. 2. Guide sheath being advanced to the target under fluoroscopic guidance.

from the BTPNA procedure was also correlated with that of the nodule from the resected lobe.

Secondary end points included parameters such as the biopsy yield, defined as the number of nodules with at least one biopsy sufficient for a tissue diagnosis divided by the number of nodules sampled. Other parameters evaluated included procedural time points, length of tunnel path created, number and adequacy of the biopsy samples.

\section{Results}

Six patients were enrolled in this study ( 3 men and 3 women), with a median age of 68 years (range $52-72$ ). The mean length of the 5 tunnels from POE on the airway wall to the nodule was $29 \mathrm{~mm}$ (range 11-46). The size of the lesions ranged from 14 to $21 \mathrm{~mm}$ in long-axis diameter, 
Table 1. Safety and procedural aspects of the BTPNA procedure

\begin{tabular}{|c|c|c|c|c|c|c|c|c|c|}
\hline LUL & 23 & yes & 8 & 8 & 1.8 & no & 46 & 20 & NSCLC \\
\hline LLL & 20 & yes & 10 & 14 & 2.5 & yes (pneumothorax) & 13 & 1 & SCC \\
\hline RUL & 15 & yes & NA & 25 & 7.5 & no & NA & 28 & Adenocarcinoma \\
\hline RLL & 17 & no & 20 & 25 & 1.3 & yes (pneumothorax) & 45 & 10 & SCLC \\
\hline
\end{tabular}

LUL = Left upper lobe; LLL = left lower lobe; RUL = right upper lobe; RLL = right lower lobe; NSCLC = non-small-cell lung cancer; SCC = small-cell carcinoma; SCLC = small-cell lung cancer; NA = not applicable.

with a mean target diameter of $17.5 \mathrm{~mm}$. Two to 5 biopsies were sampled using a forceps in each patient (mean 3.8 biopsies). Further specifications are provided in table 1 .

A positive biopsy was obtained in all 5 cases in which the BTPNA procedure could be successfully completed. There were no significant intraprocedural adverse events (table 1). Chest X-rays performed $2 \mathrm{~h}$ after the procedure revealed 2 patients who had experienced a pneumothorax. One had a maximal extent of $6 \mathrm{~mm}$ from the edge and did not need any further intervention. In the second case, a chest tube had to be inserted in order to resolve the pneumothorax. Both cases were characterized by small distances of the nodule and pleural surface ( 1 and $9 \mathrm{~mm}$ ). By contrast, tunnel length could not be attributed to the development of a pneumothorax, with one tunnel being comparatively short $(1.3 \mathrm{~cm})$ and the other one being at the end of the length scale $(4.5 \mathrm{~cm})$. There were no other complications within the 72-hour hospitalization following the BTPNA procedure.

Adequate histological sampling sufficient for a histological diagnosis was successfully attained in 5 patients. The biopsies obtained by BTPNA correlated with the final pathology in all 4 surgically resected nodules.

Due to registration issues of fluoroscopy and the Archimedes system, the procedure could not be conducted in case 4 . The software could not detect the C-arm, thus adequate guidance of the tunneling process would not have been ensured. A software update of the Archimedes system resolved potential connection problems in the following cases. Since the concerned pulmonary nodule was judged to be inaccessible by conventional bronchoscopic technics, a CT-guided transthoracic needle biopsy was performed in order to attain a histology.

\section{Discussion}

During the last decade, a vast number of novel guidance technologies have been developed with the aim of improving the diagnostic yield of the bronchoscopic approach to small pulmonary lesions. Those technologies, including endobronchial ultrasound with a guide sheath, virtual bronchoscopy and electromagnetic navigation, are all limited by their dependence on an airway leading to the lesion $[6,13]$. The BTPNA technology was developed with the intention of overcoming this constraint.

We conducted this study to evaluate the safety and feasibility of the BTPNA procedure in a standard endoscopy suite. This is the first evaluation of the technique being performed outside an operation theatre, followed by a clinical observation of at least $72 \mathrm{~h}$. This represents a major difference to the first report [12], since immediate thoracic surgery following BTPNA impeded the detection of specific postprocedural complications.

The procedure was successfully performed in 5 out of 6 patients with no significant adverse events observed at the time of the procedure. There was no evidence of pneumothoraces or significant immediate bleeding. However, a chest $\mathrm{X}$-ray, performed $2 \mathrm{~h}$ after the procedure, revealed 2 patients who had developed a pneumothorax. One subject was managed with the insertion of an intercostal drain, while in the other subject, the pneumothorax was small and did not need any further intervention. The development of these pneumothoraces may be due to the low distance of the nodules from the pleural surface. Both cases exhibited the smallest nodule-to-pleura distance of all cases. Tunnel length might play no role, with 1 case showing only a minor tunnel length of $1.3 \mathrm{~cm}$ (table 1 ). 
Due to the small case number, those observations need further clarification by bigger studies.

Our earlier report of the BTPNA procedure demonstrated a limitation when it came to sampling tissue in the apex of the lung [12]. This was attributed to the greater difficulty in controlling the angulation and orientation of the sheath with a dissection tip in the upper lobes. However, in the cases in this cohort, 3 nodules located in the upper lobes were successfully sampled.

In the fourth case, where the nodule was also located in the upper lobe, the procedure could not be carried out due to software problems affecting the interaction of the $\mathrm{C}$-arm and Archimedes software. Without synchronization, the guidance for the tunnelling process would have been inaccurate. After the correction of the software issue, the following two procedures could be carried out without further problems.

With a nodule access time averaging $18.8 \mathrm{~min}$, the procedure could be carried out 3 min faster than in our previous study [12], which implies a learning curve also confirmed by the subjective perception of our 2 operators.

In summary, it can be stated that this small study confirms previous results of the pilot study regarding the feasibility and high diagnostic yield of the BTPNA proce- dure. In all cases where the procedure could be carried out, a positive histology was attained. Yet, the formerly reported lack of adverse events could not be verified since 2 pneumothoraces were diagnosed in the postprocedural follow-up. Therefore, the procedure may provide an alternative to transthoracic needle aspiration in the diagnosis of pulmonary nodules. Since both pneumothoraces occurred in patients where the target nodule was in close proximity to the pleural surface, transthoracic needle aspiration may have been the diagnostic tool of choice. On the other hand, BTPNA might be a more appropriate choice for nodules in certain anatomical locations like centrally located nodules. In those cases, especially where two pleural planes need to be traversed, the transthoracic approach contains a high risk for the development of a pneumothorax [14]. Furthermore, the sensitivity of transthoracic needle aspiration drops with increasing distance between the nodule and the pleura [15]. BTPNA was applied in surgically fit patients only, which depicts a limitation of the study since the results cannot simply be generalized on inoperable patients.

The BTPNA procedure enhances the accessibility of peripheral nodules via the bronchoscopic route. Further research is warranted in order to better understand the advantages and disadvantages of the procedure.

\section{References}

1 Ferlay J, Shin H-R, Bray F, Forman D, Mathers C, Parkin DM: Estimates of worldwide burden of cancer in 2008: GLOBOCAN 2008. Int J Cancer 2010;127:2893-2917.

2 Jemal A, Siegel R, Xu J, Ward E: Cancer statistics, 2010. CA Cancer J Clin 2010;60:277-300.

-3 Aberle DR, Adams AM, Berg CD, Black WC, Clapp JD, Fagerstrom RM, Gareen IF, Gatsonis C, Marcus PM, Sicks JD; National Lung Screening Trial Research Team: Reduced lung-cancer mortality with low-dose computed tomographic screening. $\mathrm{N}$ Engl J Med 2011;365:395-409.

-4 Field JK, Oudkerk M, Pedersen JH, Duffy SW: Prospects for population screening and diagnosis of lung cancer. Lancet 2013;382:732741.

5 Wiener RS, Schwartz LM, Woloshin S, Welch HG: Population-based risk for complications after transthoracic needle lung biopsy of a pulmonary nodule: an analysis of discharge records. Ann Intern Med 2011;155:137-144.

-6 Baaklini WA, Reinoso MA, Gorin AB, Sharafkaneh A, Manian P: Diagnostic yield of fiberoptic bronchoscopy in evaluating soli- tary pulmonary nodules. Chest 2000;117: 1049-1054.

7 Rivera MP, Mehta AC; American College of Chest Physicians: Initial diagnosis of lung cancer: ACCP evidence-based clinical practice guidelines (2nd edition). Chest 2007; 132(suppl 3):131S-148S.

8 Gould MK, Donington J, Lynch WR, et al: Evaluation of individuals with pulmonary nodules: when is it lung cancer? Diagnosis and management of lung cancer, 3rd ed: American College of Chest Physicians evidence-based clinical practice guidelines. Chest 2013;143(suppl 5):e93S-e120S.

-9 Schreiber G, McCrory DC: Performance characteristics of different modalities for diagnosis of suspected lung cancer: summary of published evidence. Chest 2003;123(suppl 1):115S-128S.

10 Wang Memoli JS, Nietert PJ, Silvestri GA: Meta-analysis of guided bronchoscopy for the evaluation of the pulmonary nodule. Chest 2012;142:385-393.

11 Gaeta M, Pandolfo I, Volta S, et al: Bronchus sign on CT in peripheral carcinoma of the lung: value in predicting results of transbronchial biopsy. AJR Am J Roentgenol 1991;157: 1181-1185.

12 Herth FJ, Eberhardt R, Sterman D, Silvestri GA, Hoffmann H, Shah PL: Bronchoscopic transparenchymal nodule access (BTPNA): first in human trial of a novel procedure for sampling solitary pulmonary nodules. Thorax 2015;70:326-332.

13 Bandoh S, Fujita J, Tojo Y, et al: Diagnostic accuracy and safety of flexible bronchoscopy with multiplanar reconstruction images and ultrafast Papanicolaou stain: evaluating solitary pulmonary nodules. Chest 2003;124:1985-1992.

14 Heyer CM, Reichelt S, Peters SA, Walther JW, Müller K-M, Nicolas V: Computed tomography-navigated transthoracic core biopsy of pulmonary lesions: which factors affect diagnostic yield and complication rates? Acad Radiol 2008;15:1017-1026.

15 Priola AM, Priola SM, Cataldi A, et al: Accuracy of CT-guided transthoracic needle biopsy of lung lesions: factors affecting diagnostic yield. Radiol Med (Torino) 2007;112:11421159. 